\title{
The Role of Concomitant Radiation Boost in Neoadjuvant Chemoradiotherapy for Locally Advanced Rectal Cancer
}

\author{
HARUN BADAKHSHI ${ }^{1}$, MAHMOUD ISMAIL ${ }^{2}$, CHRISTOS BOSKOS $^{3}$, KUAILE ZHAO and DAVID KAUL $^{5}$ \\ ${ }^{1}$ Radiation Oncology, Ernst von Bergmann Medical Center, Potsdam, Germany; \\ ${ }^{2}$ Surgery, Charité University Hospital, Berlin, German; \\ ${ }^{3}$ Radiation Oncology, Metaxa Anticancer Hospital Piraeus, Athens, Greece; \\ ${ }^{4}$ Radiation Oncology, Fudan University Cancer Center, Shanghai, P.R. China; \\ ${ }^{5}$ Radiation Oncology, Charité University Hospital, Berlin, Germany
}

\begin{abstract}
Background/Aim: This study analyzed the impact of concomitant boost on long-term clinical outcomes in locally advanced rectal cancer. Patients and Methods: A total of 141 patients (median age $=61$ years) were treated with neoadjuvant chemoradiotherapy. Median total dose was 50.4 Gy. Forty-three patients received a concomitant boost. Concurrent chemotherapy consisted of 5-fluorouracil (5$F U)$, given as a 24-h continuous infusion. Mean follow-up was 83.7 months. Results: The 3, 5-, and 10-year overall survival (OS) rates were 91.9\%, 84.6\%, and $52.9 \%$, respectively. Recurrence-free survival (RFS) rates at 3, 5, and 10 years were $91.4 \%, 88.9 \%$, and $79.3 \%$, respectively. Metastasis-free survival (MFS) rates at 3, 5, and 10 years were $84.6 \%, 75.4 \%$, and $49.9 \%$, respectively. Overall, $9.9 \%$ of all patients achieved pathological complete response. Down-staging of T-or N-stage was achieved in $55.1 \%$ and $41.5 \%$ of patients. Multivariate analysis revealed that female $\operatorname{sex}(p=0.011)$, concomitant boost-radiotherapy $(p=0.014)$, and the presence of fewer than five positive lymph nodes $(p<0.001)$ were positive predictors of OS. Fewer than five positive lymph nodes was also a positive predictor for RFS $(p=0.019)$. Female gender $(p=0.018)$ and fewer than five positive lymph nodes $(p<0.001)$ were significant predictors for MFS. Conclusion: Our data support the efficacy of preoperative treatment for rectal cancer in terms of local outcomes. Intensified radiotherapy using a concomitant boost has a positive effect on $O S$.
\end{abstract}

Correspondence to: Harun Badakhshi, MD, Ph.D, Kanzowstrasse 11, 10439 Berlin, Federal Republic of Germany. Tel: +49 15771750701, e-mail: mailtohb@posteo.net

Key Words: Rectal cancer, concomitant boost, radiotherapy, 5-fluorouracil.
Locally advanced rectal cancer (LARC), which involves Union Internationale Contre le Cancer (UICC) stage II and III cancer, is typically treated with neoadjuvant chemoradiotherapy, followed by total mesorectal excision (TME). The specific radiotherapy regimen remains a topic of debate, in most European countries, as well as the United States, long-course radiotherapy (50.4 Gy) is the standard of care, whereas in most Scandinavian countries and the Netherlands, short-course radiotherapy $(5 \times 5$ Gy $)$ is administered. Fluorouracil (5-FU) is typically used as the agent in neoadjuvant radiochemotherapy, although capecitabine is discussed as a valid alternative (1).

The benefit of adjuvant chemotherapy (CTx) in patients with LARC remains unclear. National Cancer Centers Network guidelines recommend all LARC patients should receive adjuvant $\mathrm{CTx}$, even if they have achieved a pathological complete response (pCR). However, the The European Organisation for Research and Treatment of Cancer randomized trial 22921 did not confirm a beneficial effect for adjuvant CTx in patients with LARC after neoadjuvant chemoradiotherapy, although there was a trend towards better disease-free (DFS) and overall (OS) survival in patients with ypT0-2 disease. Patients in whom down-staging was not achieved also did not benefit from adjuvant CTx (2). Another retrospective analysis compared 566 patients with LARC with pCR after neoadjuvant radiotherapy or chemoradiotherapy, and detected a trend towards worse DFS in the patients administered adjuvant CTx (3).

Most national and international guidelines recommend adding a concomitant boost, based on a phase II MD Anderson trial of capecitabine plus concomitant boost radiotherapy in which better $\mathrm{pCR}, \mathrm{N}$-downstaging, and $\mathrm{T}$ downstaging rates were detected after chemoradiation with concomitant boost and oral capecitabine (4). Other similar trials have not confirmed these findings (5), and thus, the role of concomitant boost remains a topic of debate. 


\section{Patients and Methods}

Treatment decisions, patient selection, and treatment procedures. We performed a retrospective analysis of 141 patients who had undergone neoadjuvant chemoradiotherapy and surgery for LARC (T3/T4 with/without N+) between January 1995 and December 2009. Followup data were analyzed until March 2011. At our institution, treatment decisions are based on an interdisciplinary vote, and patients with LARC usually receive neoadjuvant CTx with 5-FU infusion, followed by surgery. In the last decade, TME and postoperative 5-FU-based CTx have become standard practice at our institution. However, since we analyzed data collected in 1995, several of this study's patients had received neoadjuvant CTx and undergone traditional surgery, which was not followed by adjuvant CTx.

Chemoradiotherapy and surgery. At our institution, radiotherapy is performed using CT-based 3D-conformal treatment planning. The clinical target volume (CTV) includes the tumor, the mesorectal tissue including the perirectal and presacral nodes, and the internal iliac lymph nodes (6). All patients are irradiated in the prone position on a belly board, using a CT-planned three-field technique with 6-MV photons and a multileaf collimator. Single fractions of $1.8 \mathrm{~Gy} / \mathrm{d}$ were administered five times a week, usually up to a total dose of $45 \mathrm{~Gy}$.

A sequential concomitant tumor bed boost of 5.4 Gy was introduced as standard care in our clinic, following the 2006 publication of the phase II MD Anderson trial of capecitabine plus concomitant boost radiotherapy (4).

Concomitant CTx consists of continuous 5-FU-infusion, and surgical resection, which is generally TME, is usually performed 56 weeks after the completion of chemoradiotherapy. For tumors located $10 \mathrm{~cm}$ above the anal verge, a partial mesorectal excision (PME) is feasible, and anterior resection, intersphincteric resection, or abdominoperineal resection may also be performed, according to clinical staging and the patient's preference. Completeness of TME is evaluated and documented by every surgeon after resection by injecting methylene blue into the superior arteria rectalis.

Adjuvant CTx usually starts 2-4 weeks after complete resection, using continuous 5-FU infusion therapy as long as there are no contraindications, such as serious impaired wound healing or anastomotic leakage.

Our current follow-up program is performed at 3, 6, 12, 18, and 24 months after surgery, and then every year for a total of 5 years. Each visit usually includes physical examination, abdominal ultrasound, rectosigmoidoscopy, and endorectal ultrasound in cases of sphinctersaving surgery. Pelvic CT (or magnetic resonance imaging) scans are performed at $3,12,24$, and 36 months after surgery. A complete colonoscopy is required at 18,36 , and 60 months after surgery.

Treatment-related toxicities are registered according to the National Cancer Institute Common Terminology Criteria for Adverse Events, version 3.0.(7).

Statistical analysis. All statistical analyses were performed using SPSS Statistics version 19 (IBM, Armonk, NY, USA). A $p$-value below 0.05 was considered significant.

\section{Results}

Patients. One hundred and forty-one patients with rectal cancer who had been treated in our Department between January 1995 to December 2009 were included in our analysis. Patient
Table I. Patient characteristics $(n=141)$

\begin{tabular}{lcc}
\hline Characteristic & & Value \\
\hline Medium age (range), years & & $61(36-79)$ \\
Gender, n (\%) & M & $89(63.1)$ \\
UICC Stage, n (\%) & F & $52(36.9)$ \\
T-Stage, n $(\%)$ & II & $60(42.6)$ \\
& III & $81(57.4)$ \\
& T1 & $1(0.7)$ \\
T-Stage, n $(\%)$ & T3 & $2(1.4)$ \\
& T4 & $115(81.6)$ \\
& n.a. & $22(15.6)$ \\
& N0 & $1(0.7)$ \\
Histology, n $(\%)$ & N1 & $34(24.1)$ \\
& N2 & $40(28.4)$ \\
& N+ & $19(13.5)$ \\
& Nx & $21(14.9)$ \\
& n.a. & $26(18.4)$ \\
& upper rectum & $1(0.7)$ \\
& middle rectum & $9(6.4)$ \\
& lower rectum & $75(53.2)$ \\
& Adenocarcinoma & $57(40.4)$ \\
& Mucinous carcinoma & $116(82.3)$ \\
& Undifferentiated carcinoma & $1(0.7)$ \\
\hline
\end{tabular}

UICC: Union Internationale Contre le Cancer; n.a.: not available.

characteristics are summarized in Table I. Median follow-up was 83.7 months, and the median patient age was 61 years (range $=36-79$ years). Of the patients included in this study, $63 \%$ were male and $37 \%$ were female. Clinical staging revealed $42.6 \%$ UICC stage II and $57.4 \%$ stage III cancers.

Chemoradiotherapy and surgery. Treatment details are summarized in Table II. The median RT dose was 50.4 Gy (range=18-59.4 Gy), and all patients received single doses of 1.8 Gy. Five patients received a total dose of 59.4 Gy instead of $50.4 \mathrm{~Gy}$, and five did not receive the full planned dose due to side-effects. In all, 139 out of 141 patients received concurrent 5-FU-based CTx by infusion; one patient refused 5-FU-based therapy. The majority of patients (134/140) received $225 \mathrm{mg} / \mathrm{m}^{2} 5-\mathrm{FU}$.

The median interval between radiotherapy termination and surgery was 5.1 weeks (range $=0.4-25.7$ weeks), and the delay was longer than 8 weeks for $8.5 \%$ of patients. Low anterior resection was performed in $73.8 \%$ of cases, abdominoperineal resection in $21.3 \%$, and intersphincteric resection in $2.8 \% ; 2.1 \%$ of patients were treated with other surgical methods.

Pathologic data. The total number of examined lymph nodes was recorded for 134 (95\%) patients and was unknown for seven $(5 \%)$ patients. The median number of examined nodes 
Table II. Treatment characteristics.

\begin{tabular}{lcc}
\hline Treatment & Dosage & Value \\
\hline Neoadjuvant RT & Single dose, & $1.8(1.8-1.8)$ \\
& $\begin{array}{c}\text { median (range), Gy } \\
\text { Total dose, }\end{array}$ & $50.4(18.0-59.4)$ \\
& median (range), Gy & \\
Neoadjuvant CTx, n (\%) & $225 \mathrm{mg} / \mathrm{m}^{2} 5$-FU & $134(95.7)$ \\
& (infusion) & \\
& $250 \mathrm{mg} / \mathrm{m}^{2} 5$-FU & $5(3.6)$ \\
(infusion) & $1(0.7)$ \\
Surgery, n (\%) & $825 \mathrm{mg} / \mathrm{m}^{2}$ CAP & $104(73.8)$ \\
& LAR & $30(21.3)$ \\
& APR & $4(2.8)$ \\
& ISR & $3(2.1)$ \\
\hline
\end{tabular}

RT: Radiotherapy; CTx:_hemotherapy; 5-FU: 5-fluouracil; CAP: capcitabine; LAR: low anterior resection; APR: abdominoperineal resection; ISR: intersphincteric resection.

was 12 . Adequate lymph node staging according to the UICC staging system (i.e. at least 12 nodes) was reported for $64.1 \%$ of patients.

Univariate analysis of survival. Univariate analysis of survival is shown in Table III. The 3-, 5- and 10-year OS rates were $91.9 \%, 84.6 \%$, and $52.9 \%$, respectively. Corresponding RFS rates were $91.4 \%, 88.9 \%$, and $79.3 \%$, respectively, and MFS rates were $84.6 \%, 75.4 \%$, and $49.9 \%$, respectively. Only $9.9 \%$ of all patients achieved pCR. Downstaging of T- or N-stage was achieved in $55.1 \%$ and $41.5 \%$ of patients, respectively.

Significantly improved OS rates correlated with female gender $(p=0.006)$, the application of a tumor bed boost $(p=0.008)$, lower ypN-stage $(p<0.001)$, and a lower number of positive lymph nodes $(\mathrm{n}=0-4$ vs. $\mathrm{n}>4, \quad p<0.001)$. Significantly improved RFS correlated with lower ypN-stage $(p=0.002)$. Significantly improved MFS correlated with female gender $(p=0.027)$, lower ypN-stage $(p<0.001)$, and a lower number of positive lymph nodes $(n=0-4$ vs. $n>4$, $p<0.001)$. There was a trend towards improved MFS in patients who had received a tumor bed boost $(p=0.06)$.

Multivariate analysis of survival. Multivariate analysis (Table III) revealed that female gender $(p=0.011)$, concomitant boost-radiotherapy $(p=0.014)$, and fewer than five positive lymph nodes $(p<0.001)$ were predictors for improved OS. Fewer than five positive lymph nodes was also a significant positive predictor for RFS $(p=0.019)$. Female gender $(p=0.018)$ and fewer than five positive lymph nodes $(p<0.001)$ were both significant predictors for improved MFS. Age, UICC stage, cT3 vs. other cT-stages,
Table III. Impact of different clinical and pathological factors on overall $(O S)$, recurrence-free (RFS) and metastasis-free (MFS) survival.

\begin{tabular}{|c|c|c|c|c|c|c|c|}
\hline \multirow[b]{3}{*}{ Characterstic } & \multirow[b]{3}{*}{$\mathrm{n}$} & \multicolumn{6}{|c|}{$p$-Value } \\
\hline & & \multicolumn{3}{|c|}{ Univariate analysis } & \multicolumn{3}{|c|}{ Multivariate analysis } \\
\hline & & OS & RFS & MFS & OS & RFS & MFS \\
\hline \multicolumn{8}{|l|}{ Age } \\
\hline$>60$ Years & 77 & $\mathrm{~ns}$ & $\mathrm{~ns}$ & $\mathrm{~ns}$ & ns & $\mathrm{ns}$ & $\mathrm{ns}$ \\
\hline$\leq 60$ Years & 64 & & & & & & \\
\hline \multicolumn{8}{|l|}{ Gender } \\
\hline M & 89 & 0.006 & $\mathrm{~ns}$ & 0.02 & 0.01 & $\mathrm{~ns}$ & 0.018 \\
\hline $\mathrm{F}$ & 52 & & & & & & \\
\hline \multicolumn{8}{|l|}{ UICC } \\
\hline II & 82 & ns & $\mathrm{ns}$ & ns & $\mathrm{ns}$ & $\mathrm{ns}$ & ns \\
\hline III & 59 & & & & & & \\
\hline \multicolumn{8}{|l|}{ cT3 } \\
\hline cT3 & 115 & $\mathrm{~ns}$ & ns & ns & ns & $\mathrm{ns}$ & ns \\
\hline Other & 26 & & & & & & \\
\hline \multicolumn{8}{|l|}{ Boost } \\
\hline Yes & 43 & 0.008 & ns & ns $(0.06)$ & 0.014 & ns & ns \\
\hline No & 98 & & & & & & \\
\hline \multicolumn{8}{|l|}{ TME } \\
\hline Yes & 41 & $\mathrm{~ns}$ & ns & ns & ns & ns & $\mathrm{ns}$ \\
\hline No & 100 & & & & & & \\
\hline \multicolumn{8}{|l|}{$\mathrm{pN}(\mathrm{n})$} \\
\hline $0-4$ & 131 & $<0.001$ & ns & $<0.001$ & $<0.001$ & 0.019 & $<0.001$ \\
\hline$>4$ & 10 & & & & & & \\
\hline \multicolumn{8}{|l|}{ ypN } \\
\hline ypNO & 83 & $<0.001$ & 0.002 & $<0.001$ & ns & ns & $\mathrm{ns}$ \\
\hline ypN1 & 29 & & & & & & \\
\hline ypN2 & 18 & & & & & & \\
\hline \multicolumn{8}{|c|}{ Adjuvant CTx } \\
\hline Yes & & ns & ns & ns & $\mathrm{ns}$ & $\mathrm{ns}$ & $\mathrm{ns}$ \\
\hline No & & & & & & & \\
\hline
\end{tabular}

UICC: Union Internationale Contre le Cancer; M: male; F: female; TME: total mesorectal excision; CTx: chemotherapy; $\mathrm{pN}(\mathrm{n})$ : number of pathological metastatic lymph nodes; ns: not significant.

TME, pCR, and adjuvant CTx were not significantly correlated with a change in OS, RFS, or MFS rates.

Concomitant boost was the only therapy-associated factor with a significant effect on OS in both univariate and multivariate analyses. No significant difference in UICC stage, T-stage, or $\mathrm{N}$-stage was observed between patients who had received concomitant boost and patients who had received standard radiotherapy.

Acute toxicity. Overall, treatment was well tolerated. Fifty-six (39.7\%) patients developed grade I gastrointestinal toxicity, and $17(12.1 \%)$ developed grade II. No acute grade III or grade IV gastrointestinal toxicity was observed. Thirty-five (24.8\%) patients developed grade I acute genitourinary toxicity, and four patients $(2.8 \%)$ developed grade II. No acute 
grade III or grade IV genitourinary toxicity was observed.

The rate of grade II or higher acute toxicity among patients who had received a boost and those who had received standard therapy was not significantly different.

Chronic toxicity. Fifteen (10.6\%) patients developed grade I gastrointestinal toxicity, and two (1.4\%) developed chronic grade II gastrointestinal toxicity. Fifteen patients (10.6\%) developed chronic grade III gastrointestinal toxicity. Nine (6.4\%) patients developed chronic grade I genitourinary toxicity, and one $(0.7 \%)$ developed chronic grade II genitourinary toxicity. No chronic grade III or IV genitourinary toxicity was observed. The rate of grade II or higher chronic toxicity among patients who had received a boost and those who had received standard therapy was not significantly different.

\section{Discussion}

The incidence of rectal cancer is $15-25 / 100,000$ per year in European countries, a number that represents approximately $35 \%$ of all colorectal malignancies. The optimal neoadjuvant treatment for LARC is still a topic of debate. In the past decades, OS has increased significantly. The current standard treatment is preoperative chemoradiotherapy followed by TME and adjuvant CTx. However, several aspects of this standard are under debate. Questions remain regarding the meaningfulness of adding a boost in neoadjuvant radiotherapy, the optimal treatment for stage T3 rectal cancer, and whether postoperative CTx is truly beneficial.

In this study, the 3-, 5-, and 10-year OS rates were $91.9 \%$, $84.6 \%$, and $52.9 \%$, respectively, and the corresponding RFS rates were $91.4 \%, 88.9 \%$, and $79.3 \%$, respectively. Osti et al. reported a slightly lower 3-year OS $(86.8 \%, \mathrm{n}=65)$ and 3year RFS $(81 \%)$ rates in patients with LARC treated with neoadjuvant chemoradiotherapy using capecitabine and concomitant boost (8), while Hernando-Requejo et al. reported a slightly higher 3-year OS $(95.4 \%, n=74)$ and 3year RFS (95.4\%) rates in patients with LARC treated with the same neoadjuvant chemoradiotherapy (9).

We found an acceptable toxicity profile in the current study, with non-hematological severe toxicities (grade 3 or more) observed in $10.6 \%$ of patients, and no grade 4 toxicity observed. Other studies have shown similar rates of severe toxicity during concomitant boost radiotherapy and concurrent fluoropyrimidine-based CTx (10). Kim et al. also reported severe (grade 3-4) non-hematological toxicities in $15.8 \%$ of patients $(n=38)$ who had received concomitant boost radiotherapy $(43.2 \mathrm{~Gy} / 24$ fractions plus boost 7.2 Gy/12 fractions) and associated bolus 5-FU (5). Osti et al. also reported non-hematological severe toxicities (grade 3 or more) in $15 \%$ of their patients $(n=65)(8)$.
In this study, $9.9 \%$ of all patients achieved pCR, although this number is slightly lower than that those of other studies. For example, Vestermark et al. reported a pCR rate of $13 \%$ $(n=52)$ in a phase II trial of high-dose radiotherapy (60 Gy) and Tegafur/uracil and leucovorin in patients with LARC (9). In addition, Osti et al. reported a pCR rate of $17 \%(n=65)$ in patients with LARC treated with neoadjuvant chemoradiotherapy using capecitabine and concomitant boost (7). Finally, Caravatta et al. described a pCR rate of $32 \% \quad(n=25)$ in patients treated with preoperative concomitant boost radiotherapy (55 Gy/5 weeks) and concurrent CTx with raltitrexed plus oxaliplatin (11). However, each of these studies used different CTx agents, which makes inter-study comparisons difficult.

In this study, down-staging of $\mathrm{T}$ - or $\mathrm{N}$-stage was achieved in $55.1 \%$ and $41.5 \%$ of patients, respectively, and these results are comparable to those found in the phase II MD Anderson trial of capecitabine plus concomitant boost radiotherapy (namely $51 \%$ and $52 \%$, respectively (4). Osti et al. also reported similar numbers in patients with LARC treated with capecitabine and concomitant boost, with T-stage and N-stage downstaging achieved in $40 \%$ and $58 \%(n=65)$ of patients, respectively (8). Kim et al. reported corresponding rates of $41.7 \%$ and $85.2 \%(n=38)$, respectively, in patients with LARC who had received neoadjuvant chemoradiotherapy plus concomitant boost and two cycles of bolus 5-FU and leucovorin (5).

Boost. Most guidelines recommend that LARC be treated with adjuvant chemoradiotherapy plus boost. These recommendations are mainly based on the phase II MD Anderson trial of capecitabine plus concomitant boost radiotherapy that reported $\mathrm{pCR}, \mathrm{N}$-, and T-downstaging of $18 \%, 52 \%$, and $51 \%$, respectively, after chemoradiation with concomitant boost (52.5 Gy/30 fractions) plus oral capecitabine (4). Other studies have confirmed the positive effects of neoadjuvant chemoradiation with concomitant boost radiotherapy in terms of local outcomes $(8,10)$. One such study is the Madrid study of pCR in LARC after preoperative intensity modulated radiotherapy plus integrated-boost chemoradiation, which found higher rates of ypCR and pT down-staging (9).

However, other reports have described contradictory results, such as the prospective phase II KROG 04-01 trial, where the authors found that concomitant boost irradiation did not improve down-staging rates, tumor regression grades, or tumor volume reduction rates (5). In the long-term followup, the authors reported that concomitant small-field boost irradiation for 5 weeks in patients with rectal cancer was non-superior to conventional irradiation (6 weeks) in terms of both tumor response and survival (12).

In the present study, concomitant boost was the only therapy-associated factor with a significant effect on OS in 
both univariate and multivariate analyses. However, the rate of acute or chronic grade II or higher toxicity in patients who had received a boost and those who had received standard therapy was not significantly different. Moreover, the rate of pCR was not significantly higher in patients who had received a concomitant boost. A possible explanation might be the relatively small number of patients with pCR in the overall patient population (14 out of 141 patients) makes statistical analysis of factors influencing pCR problematic. Furthermore, it is striking that even though concomitant boost was a significant factor for OS, the impact on RFS and MFS was not significant. However, there was a trend towards improved MFS rates in the boost group after univariate analysis, although this trend was not confirmed by multivariate analysis. Apart from the inherent limitations of a retrospective study, we can only speculate that the group of patients that received a boost $(n=43)$ was too small to detect a significant change in the MFS and RFS.

\section{Conclusion}

Our data support the efficacy of preoperative treatment for rectal cancer in terms of local outcomes. Intensified radiotherapy using a concomitant boost has a positive effect on OS. Prospective studies with longer follow-up are needed to determine the efficacy of this treatment.

\section{Conflicts of Interests}

The Authors declare no conflict of interest in regard to this study.

\section{References}

1 Hofheinz RD, Wenz F, Post S, Matzdorff A, Laechelt S, Hartmann JT, Muller L, Link H, Moehler M, Kettner E, Fritz E, Hieber U, Lindemann HW, Grunewald M, Kremers S, Constantin C, Hipp M, Hartung G, Gencer D, Kienle P, Burkholder I and Hochhaus A: Chemoradiotherapy with capecitabine versus fluorouracil for locally advanced rectal cancer: a randomised, multicentre, non-inferiority, phase 3 trial. Lancet Oncology 13(6): 579-588, 2012.

2 Collette L, Bosset JF, den Dulk M, Nguyen F, Mineur L, Maingon P, Radosevic-Jelic L, Pierart M and Calais G Patients with curative resection of cT3-4 rectal cancer after preoperative radiotherapy or radiochemotherapy: Does anybody benefit from adjuvant fluorouracil-based chemotherapy? A trial of the European Organisation for Research and Treatment of Cancer Radiation Oncology Group. J Clin Oncol 25(28): 4379-4386, 2007.

3 Capirci C, Valentini V, Cionini L, De Paoli A, Rodel C, GlynneJones R, Coco C, Romano M, Mantello G, Palazzi S, Mattia FO, Friso ML, Genovesi D, Vidali C, Gambacorta MA, Buffoli A, Lupattelli M, Favretto MS and La Torre G: Prognostic value of pathologic complete response after neoadjuvant therapy in locally advanced rectal cancer: long-term analysis of 566 ypCR patients. Int J Radiat Oncol Biol Phys 72(1): 99-107, 2008.
4 Krishnan S, Janjan NA, Skibber JM, Rodriguez-Bigas MA and Wolff RA, Das P, Delclos ME, Chang GJ, Hoff PM, Eng C, Brown TD, Crane CH, Feig BW, Morris J, Vadhan-Raj S and Hamilton SR and Lin EH: Phase II study of capecitabine (Xeloda) and concomitant boost radiotherapy in patients with locally advanced rectal cancer. Int J Radiat Oncol Biol Phys 66(3): 762-771, 2006.

5 Kim DY, Kim TH, Jung KH, Chang HJ, Lim SB, Choi HS, Jeong SY, Nam TK, Ryu SY, Lee DS, Choi SI, Kang JH and Yoon SC: Preoperative chemoradiotherapy with concomitant small field boost irradiation for locally advanced rectal cancer: a multi-institutional phase II study (KROG 04-01). Dis Colon Rectum 49(11): 1684-1691, 2006.

6 Siegel R, Burock S, Wernecke KD, Kretzschmar A, Dietel M, Loy V, Koswig S, Budach V and Schlag PM: Preoperative shortcourse radiotherapy versus combined radiochemotherapy in locally advanced rectal cancer: a multi-centre prospectively randomised study of the Berlin Cancer Society. BMC Cancer 9:50, 2009 .

7 https://ctep.cancer.gov/protocoldevelopment/electronic_ applications/docs/ctcaev3.pdf (Last accessed April 17, 2017)

8 Osti MF, Agolli L, Bracci S, Masoni L, Valeriani M, Falco T, De Sanctis V and Maurizi Enrici R: Neoadjuvant chemoradiation with concomitant boost radiotherapy associated to capecitabine in rectal cancer patients. Int J Colorectal Dis 29(7): 835-842, 2014.

9 Hernando-Requejo O and Lopez M, Cubillo A, Rodriguez A, Ciervide R, Valero J, Sanchez E, Garcia-Aranda M and Rodriguez J, Potdevin G and Rubio C: Complete pathological responses in locally advanced rectal cancer after preoperative IMRT and integrated-boost chemoradiation. Strahlen Onkol 190(6): 515-520, 2014.

10 Vestermark LW, Jacobsen A, Qvortrup C, Hansen F, Bisgaard C, Baatrup G, Rasmussen P and Pfeiffer P: Long-term results of a phase II trial of high-dose radiotherapy (60 Gy) and UFT/1leucovorin in patients with non-resectable locally advanced rectal cancer (LARC). Acta Oncol 47(3): 428-433, 2008.

11 Caravatta L, Padula GD, Picardi V, Macchia G, Deodato F, Massaccesi M, Sofo L, Pacelli F, Rotondi F, Cecere G, Sallustio G, Di Lullo L, Piscopo A, Mignogna S, Bonomo P, Cellini N, Valentini V and Morganti AG: Concomitant boost radiotherapy and multidrug chemotherapy in the neoadjuvant treatment of locally advanced rectal cancer: results of a phase II study. Acta Oncol 50(8): 1151-1157, 2011.

12 Lee JH, Kim DY, Nam TK, Yoon SC, Lee DS, Park JW, Oh JH, Chang HJ, Yoon MS, Jeong JU and Jang HS: Long-term followup of preoperative pelvic radiation therapy and concomitant boost irradiation in locally advanced rectal cancer patients: a multi-institutional phase II study (KROG 04-01). Int J Radiat Oncol Biol Phys 84(4): 955-961, 2012.
Received April 8, 2017

Revised April 18, 2017

Accepted April 25, 2017 